\title{
Síndrome de Burnout y Satisfacción Laboral en Docentes de Nivel Secundario
}

\author{
BURNOUT SYNDROME AND JOB SATISFACTION IN SECONDARY SCHOOL TEACHERS
}

Nancy Marlene Malander

Docente de nivel Superior. Profesora universitaria en Ciencias Económicas. Instituto Superior Adventista de Misiones, Misiones, Argentina.

\section{RESUMEN}

Los docentes ejercen una actividad considerada estresante y proclive a desencadenar el síndrome de burnout. Esta investigación pretendió determinar si la satisfacción laboral y algunas características sociodemográficas y laborales de los docentes de nivel secundario, pueden atenuar o bien potenciar el síndrome. Participaron 123 docentes de seis colegios de gestión privada de la provincia de Misiones, Argentina. Se utilizó el Inventario de Burnout de Maslach y Jackson ${ }^{1}$; la Escala de Satisfacción Laboral para Docentes, de Oros y Main ${ }^{2}$, e indicadores de variables sociodemográficas y laborales. El procedimiento estadístico utilizado fue la Regresión lineal múltiple. Los resultados mostraron que la baja satisfacción laboral predice significativamente el burnout y cada una de sus dimensiones. Los factores intrínsecos de la satisfacción laboral mostraron ser los mejores predictores del cansancio emocional, la despersonalización y la realización personal. Los factores extrínsecos predicen significativamente sólo la dimensión de cansancio emocional. Los individuos más satisfechos con su tarea, con mayor motivación y autorrealización son menos propensos a sufrir y desarrollar el Síndrome. La única variable sociodemográfica y laboral que predijo significativamente el burnout, fue la edad. Los docentes más jóvenes mostraron mayor predisposición a padecer el Síndrome.

(Malander N, 2016. Síndrome de Burnout y Satisfacción Laboral en Docentes de Nivel Secundario. Cienc Trab. Sep-Dic; 18 [57]: 177-182).

Palabras clave: BURNOUT, SATISFACCIÓN LABORAL, DOCENTES.

\section{ABSTRACT}

Teachers practise an activity considered stressful and inclined to trigger the burnout syndrome. This research aimed to determine if the job satisfaction and some sociodemographic and work characteristics of teachers in high school, can attenuate or promote the syndrome. Participants were 123 teachers from six privately run schools in the province of Misiones, Argentina. The Burnout Inventory of Maslach and Jackson (1997) was used; The Occupational Satisfaction Scale for Teachers, by Oros and Main (2004), and indicators of socio-demographic and labor variables. The statistical procedure used was the Multiple Linear Regression. The results showed that the low job satisfaction predicts significantly the burnout and each of its dimensions. The intrinsic factors of job satisfaction were the best predictors of emotional exhaustion, depersonalization and personal fulfillment. Extrinsic factors predict significantly only the dimension of emotional exhaustion. Individuals more satisfied with their task, with greater motivation and self-actualization are less likely to suffer and develop the Syndrome. The only sociodemographic and labor variable that significantly predicted burnout was age. Younger teachers showed a greater predisposition to suffer the Syndrome.

Keywords: BURNOUT, JOB SATISFACTION, TEACHERS.

\section{INTRODUCCIÓN}

En todo proceso de enseñanza-aprendizaje existen dos sujetos claves, el que enseña y el que aprende. Para que dicho proceso tenga éxito y se logre un aprendizaje significativo y de calidad es fundamental que ambos sujetos gocen de cierta salud física, psíquica y emocional que les permita interactuar y progresar de una manera apropiada. Para que el docente pueda realizar su tarea

Correspondencia / Correspondence:

Nancy Malander.

Calle México 620. ISAM. Código Postal 3315, Leandro N. Alem,

Misiones, Argentina

Tel.: 3754440178

e-mail: marlenemalander@gmail.com

Recibido: 11 de Octubre de 2016 / Aceptado: 25 de Noviembre de 2016

con eficacia es necesario que se sienta a gusto con la misma, que la perciba útil, y que cuente con las competencias, habilidades y capacidades necesarias; sin embargo, dentro de las problemáticas que han aquejado al sector del profesorado en las últimas décadas, ha cobrado relevancia el aumento de la prevalencia del síndrome de burnout y la disminución de la satisfacción laboral, mermando por consiguiente la salud y las capacidades de los afectados.

La mayoría de los docentes que inician su carrera profesional lo hacen con altas expectativas acerca de los resultados que obtendrán de su tarea. Los docentes jóvenes y recién graduados llegan al aula con nuevas metodologías, conocimientos y competencias adquiridas durante el cursado de la carrera y grandes perspectivas de contribuir para lograr cambios y mejoras en la tarea docente y, sobre todo, resultados positivos e importantes en cada uno de los alumnos con los que se relacionarán durante su trayectoria profesional. Pero en muchos casos, dichas expectativas no se cumplen, lo que puede dar lugar a una sensación de fracaso, a un estado de insatisfacción laboral que se traduce en licencias por diversos motivos, tanto físicos 
como psicológicos, y a un desgaste profesional que, si no se logra revertir, puede culminar en un estado generalizado de frustración, estrés crónico y, en algunos casos, en un Síndrome que ha sido denominado como síndrome de burnout o; en español, "Síndrome de Quemarse por el Trabajo" (en adelante SQT). ${ }^{3}$

La tarea de los docentes los lleva a implicarse durante muchas horas al día en los problemas, dificultades, preocupaciones y necesidades de sus alumnos e incluso en los problemas que enfrentan los padres y familias de sus alumnos, en los cuales no siempre pueden contribuir para su solución. Además, las familias y la sociedad se han vuelto incapaces de cumplir con las responsabilidades que les tocan en la formación de los niños y jóvenes, por lo que van sumando demandas a la cada vez más compleja tarea docente. El contexto escolar y la demanda de estos nuevos roles resultantes de la delegación de responsabilidades hacen que el docente se sienta frustrado cuando descubre que no puede solucionar todas las problemáticas que se le presentan, superando dichas demandas sus posibilidades. Esta realidad genera un desgaste personal importante, que puede conducir al estrés crónico, al desgaste emocional e incluso al síndrome de burnout. ${ }^{4}$ Una de las definiciones más aceptadas de burnout es la elaborada por Maslach y Jackson ${ }^{5}$, que hace referencia a que es un síndrome compuesto por tres dimensiones. La primera está constituida por un estado de agotamiento emocional y cinismo que lleva a que el sujeto sienta que no es capaz de dar más de sí (Cansancio emocional). Dicho agotamiento es acompañado de manifestaciones somáticas y psicológicas como el abatimiento, la ansiedad y la irritabilidad. Generalmente constituye la primera fase del proceso y presenta una progresiva pérdida de las energías vitales, y una desproporción cada vez más significativo entre el trabajo realizado y el cansancio experimentado, lo que lleva a las personas a dejar de disfrutar de las tareas que realizan. En esta fase, dichos sujetos son generalmente catalogados como quejosos, insatisfechos e irritables por sus compañeros y superiores.

En segundo lugar, también genera actitudes y sentimientos negativos hacia los clientes o usuarios de la tarea y hacia los colegas (Despersonalización). Los sujetos que padecen el síndrome presentan una actitud impersonal, con aislamiento de los demás y con intentos de culpabilizarlos de la frustración y el fracaso que sienten en su trabajo. Es un modo de responder a los sentimientos de impotencia, indefensión y desesperanza personal.

Un tercer aspecto está dado por la tendencia que manifiesta el profesional a evaluarse negativamente a sí mismo y a su tarea (Baja realización personal). En esta fase el sujeto se retira de todas las actividades que no sean las laborales. Hay pérdida de ideales y un creciente alejamiento de actividades familiares, sociales y recreativas. $^{5}$

Es preciso analizar aquellos factores que pueden predisponer la aparición de este síndrome, con el objeto de poder revertir o minimizar su aparición dentro de las instituciones educativas. Es muy difícil atribuir la aparición del síndrome a un solo factor, ya que se trata de un problema complejo cuya aparición es multicausal; sin embargo, en base a varios estudios realizados, entre las variables que podrían repercutir sobre la prevalencia del sindrome de burnout en los docentes se encuentran la satisfacción laboral y ciertos factores sociodemográficos y laborales, como la edad, el género, la paternidad, el estado civil, la antigüedad laboral, la carga horaria, entre otras.

La satisfacción laboral es definida en general como una actitud y experiencia emocional positiva. Salazar ${ }^{6}$ la define como un conjunto de actitudes positivas que el trabajador adopta hacia su labor, mientras que la insatisfacción hace referencia a las actitudes negativas que van acompañadas de pérdida de identificación psicológica y emocional hacia el trabajo, definición adoptada por esta investigación. Oros y Main ${ }^{2}$ consideran que toda actitud se desarrolla a partir de una interacción entre dos partes, en este caso entre el trabajador y las condiciones laborales. Si estas son percibidas de manera positiva, el trabajador adoptará de forma natural una actitud favorable y su conducta será un reflejo de esta. Las autoras afirman que aceptar esta premisa implica preguntarse qué aspectos del entorno laboral promueven actitudes positivas y cuáles se asocian con la insatisfacción.

El cuanto a la satisfacción laboral docente, su estudio ha adquirido mucha importancia en los últimos años, en gran medida debido al impacto que puede tener en la salud física y psicológica de los integrantes de todo tipo de organizaciones. Además, desde un concepto de calidad total, la institución escolar también debe preocuparse por el desarrollo profesional y personal de sus docentes, que a su vez se verá reflejado en una mayor calidad educativa. Un docente con alto nivel de satisfacción laboral estará en mejores condiciones de salud física y psicológica, lo que redundará en una mayor eficiencia y eficacia de su tarea, disminuirá su frustración y mejorará su afrontamiento del estrés, evitando en muchos casos el estrés crónico. ${ }^{7,8}$

Según Parada et $\mathrm{al}^{9}$, el burnout tiene muchos factores desencadenantes; sin embargo, la disminución de la satisfacción laboral es uno de los predictores más importantes. Garnier y Lee $^{9}$ sostienen que cuanto menor sean los niveles de satisfacción laboral, mayor será la prevalencia del síndrome, específicamente de la dimensión de agotamiento emocional. Hermosa Rodríguez ${ }^{10}$ partió de la hipótesis de que la satisfacción laboral es un antecedente del síndrome y encontró correlaciones negativas entre satisfacción laboral y burnout; y Cavalcante Silva ${ }^{11}$ postula que el burnout es una variable resultante de la satisfacción laboral no alcanzada.

Sin embargo, no existe acuerdo entre los autores acerca de si la satisfacción laboral es causa o efecto del síndrome, por lo que la presente investigación se planteó el objetivo de determinar si la satisfacción laboral y algunas variables sociodemográficas y laborales constituyen un antecedente o predictor del síndrome de burnout.

\section{MATERIALES Y MÉTODOS}

Se realizó un estudio de tipo cuantitativo, descriptivo-correlacional, y transversal.

\section{Participantes}

Se censó a un total de 133 docentes de seis instituciones de nivel secundario de gestión privada de la provincia de Misiones, Argentina, obteniendo un nivel de respuesta del 92,48\% $(\mathrm{N}=123)$. En cuanto a la variable sexo, el 59,3\% de la muestra era de sexo femenino. Las edades oscilaron entre los 23 y los 63 años $(M=$ 38,$19 ; \mathrm{DE}=8,62$ ). En cuanto al estado civil, la mayoría eran casados ( $\mathrm{n}=99 ; 80,5 \%)$, y tenían hijos ( $\mathrm{n}=89 ; 72,4 \%)$. El rango de antigüedad laboral que predominó era menor a 5 años $(n=41$; $33,3 \%)$, y el de carga horaria, era de 22 a 42 horas ( $n=79 ; 64,2 \%)$.

\section{Instrumentos}

De acuerdo con los objetivos de la presente investigación, se optó por utilizar instrumentos ya validados en Argentina: (a) el Maslach 
Burnout Inventory (MBI)1; (b) la Escala de Satisfacción Laboral para Docentes (ESLA). ${ }^{2}$

El Maslach Burnout Inventory fue desarrollado por Maslach y Jackson $^{5}$ para medir el sindrome en profesionales que prestan servicios a otras personas, resultando del análisis tres dimensiones: agotamiento emocional, despersonalización y realización personal. Es un inventario que puede ser autoadministrado, constituido por 22 items en forma de afirmaciones. La dimensión cansancio emocional (CE) está constituida por 9 ítems, que describen los sentimientos de una persona emocionalmente exhausta por las demandas de su trabajo, que superan su posibilidad de respuesta; la dimensión despersonalización (DP), por 5 ítems que describen una respuesta emocional fría hacia los receptores o beneficiarios del servicio que se presta, en el caso de la docencia, hacia los alumnos; y la dimensión realización personal (RP) quedó constituida por 8 ítems, evalúa los sentimientos de competencia y éxito en el propio trabajo, así como el logro de los objetivos personales. Dicho instrumento fue sometido al procedimiento de confiabilidad que se estableció mediante el Alpha de Cronbach, obteniendo un valor de 0,90 para la dimensión cansancio emocional, 0,79 para despersonalización y 0,71 en realización personal. ${ }^{12}$

El instrumento utilizado para medir la satisfacción laboral fue elaborado por Oros y Main ${ }^{2}$ y consta de cuatro subescalas, que se agrupan en dos tipos de factores: 1-Factores extrínsecos: que incluyen la dimensión de la tarea y la dimensión socio-organizativa y 2-Factores intrínsecos: que incluyen la dimensión motivacional y la dimensión de autorrealización. Para la construcción del instrumento las autoras tomaron como base la teoría de Herzberg, quien sostiene que la presencia de los factores extrínsecos e intrínsecos está asociada a la satisfacción laboral. ${ }^{13} \mathrm{La}$ escala consiste en una lista de 33 reactivos, escalados según el método Likert, frente a los cuales el sujeto debe responder con una de las siguientes opciones: fuerte acuerdo, acuerdo, neutro, desacuerdo, fuerte desacuerdo. Estos reactivos fueron redactados con un sentido general, pudiendo aplicarse a diversas áreas de trabajo; sin embargo, varios de ellos fueron adaptados específicamente para medir la satisfacción laboral en docentes. Con respecto a las características psicométricas, Oros y Main ${ }^{2}$ (2004) encontraron un Alfa de Cronbach para la totalidad de los items que conforman el instrumento que se situó en un valor de 0,88 . El índice de consistencia interna para el factor extrínseco fue de 0,85 y para el factor intrínseco fue de 0,77. La consistencia interna de las dimensiones es de 0,69 para la subescala tarea; 0,86 para la subescala socio-organizativa; 0,68 para motivación; y 0,74 para autorrealización.

\section{Procedimientos de recolección y análisis de datos}

En primer lugar se contactó a los directivos de los colegios secundarios de gestión privada que componen la muestra, con el fin de gestionar la autorización para la aplicación de los instrumentos entre los docentes de dichos establecimientos.

En una segunda instancia, los instrumentos fueron administrados en ocasión de reuniones de docentes del nivel secundario. Previamente a la aplicación de los instrumentos se aclaró a los docentes que la participación era completamente libre, voluntaria y anónima.

Una vez finalizada la administración de los protocolos a los docentes, se codificaron los datos y se ingresaron al programa Statistical Package for the Social Sciences (SPSS) para Windows. Para analizar la relación y poder predictivo de las variables socio- demográficas y de la satisfacción laboral sobre el burnout se realizaron análisis de correlación de Pearson y de regresión lineal múltiple, utilizando un nivel de significación p 0,05 para la interpretación de los resultados.

\section{RESULTADOS}

Satisfacción laboral y sindrome de burnout. En un primer modelo de regresión se incluyeron como predictores los factores intrínsecos y extrínsecos, y como variable dependiente el puntaje bruto de burnout, encontrándose que los factores de la satisfacción laboral predicen significativamente el burnout, $F(2,120)=36,46$, $p=0,000$. La variancia explicada estuvo en torno al $38 \%$. Se encontró que son los factores intrínsecos los que mejor predicen la variabilidad del burnout $(\beta=-0,548 ; p=0,000)$.

Un segundo análisis de regresión mostró que los factores intrínsecos y extrínsecos, explican el 36,5\% de la variabilidad de la dimensión cansancio emocional, $F(2,120)=34,55, p=0,000$; el $20 \%$ de la dimensión despersonalización, $F(2,120)=14,78, p=$ 0,000 ; y el 27,2\% de la realización personal, $F(2,120)=22,38, p$ $=0,000$. Nuevamente, son los factores intrínsecos los que muestran el mayor poder predictivo sobre las dimensiones de cansancio emocional ( $\beta=-380 ; p=0,000)$; despersonalización $(\beta=-0,444$; $p=0,000)$ y realización personal $(\beta=0,582 ; p=0,000)$ del burnout (ver Tabla 1). A mayor satisfacción intrínseca, menor es el cansancio emocional y la despersonalización y mayores los valores de realización personal.

Tabla 1.

Resumen de análisis de regresión múltiple para los factores extrínsecos e intrínsecos de la satisfacción laboral y el síndrome de burnout.

\begin{tabular}{|c|c|c|c|c|}
\hline $\begin{array}{l}\text { Factores de } \\
\text { la satisfacción }\end{array}$ & $\begin{array}{l}\text { Dimensiones } \\
\text { de burnout }\end{array}$ & B & $\beta$ & IC 95\% \\
\hline $\begin{array}{l}\text { Extrinsecos } \\
\text { Intrinsecos }\end{array}$ & $\begin{array}{l}\text { Burnout } \\
\text { Burnout }\end{array}$ & $\begin{array}{l}-0,191 \\
-1,527\end{array}$ & $\begin{array}{l}-0,099 \\
-0,548^{* * *}\end{array}$ & $\begin{array}{r}{[-0,54,0,16]} \\
{[-2,04,-1,02]}\end{array}$ \\
\hline Extrínsecos & $\begin{array}{l}\text { Cansancio emocional } \\
\text { Despersonalización } \\
\text { Realización personal }\end{array}$ & $\begin{array}{l}-0,62 \\
-0,001 \\
-0,062\end{array}$ & $\begin{array}{l}-0,288^{* *} \\
-0,001 \\
-0,108\end{array}$ & $\begin{array}{r}{[-0,43,-0,09]} \\
{[-0,17,0,17]} \\
{[-0,18,0,05]}\end{array}$ \\
\hline Intrínsecos & $\begin{array}{l}\text { Cansancio emocional } \\
\text { Despersonalización } \\
\text { Realización personal }\end{array}$ & $\begin{array}{c}-0,498 \\
-0,527 \\
0,484\end{array}$ & $\begin{array}{l}-0,380^{* * *} \\
-0,444^{* * *} \\
0,582^{* * *}\end{array}$ & $\begin{array}{r}{[-0,74,-0,25]} \\
{[-0,77,-0,28]} \\
{[0,32,0,65]}\end{array}$ \\
\hline
\end{tabular}

Con el objeto de analizar las diferentes dimensiones de la satisfacción laboral en relación al Síndrome, se ejecutó otro modelo de regresión en el que se incluyeron como predictores las dos dimensiones de los factores extrínsecos (tarea y socioorganizativa) y las dos dimensiones de los factores intrínsecos (motivación y autorrealización), y como variable dependiente el puntaje bruto de burnout, encontrándose que las dimensiones de los factores de la satisfacción laboral predicen significativamente el burnout, $F$ $(4,118)=19,60, p=0,000$. El valor del coeficiente de determinación $\mathrm{R}^{2}$ indicó que las cuatro dimensiones de la satisfacción laboral explican un 40\% del síndrome. Se encontró que la autorrealización $(\beta=-0,345 ; p=0,000)$ y la motivación $(\beta=-0,289 ; p=0,002)$ son los mejores predictores de la variabilidad del burnout, seguidos por la dimensión tarea $(\beta=-0,187 ; p=0,023)$.

Un siguiente análisis mostró que la dimensión tarea es la que mejor predice el cansancio emocional ( $\beta=-0,369 ; p=0,000)$, que la dimensión socioorganizativa predice la realización personal ( $\beta$ $=-0,204 ; p=0,037$ ), que la motivación es el mejor predictor de la 
dimensión despersonalización ( $\beta=-0,322 ; p=0,002)$, y que la autorrealización es el mayor predictor de la realización personal ( $\beta$ $=0,404 ; p=0,000$ ) (véase Tabla 2). La relación entre tareacansancio emocional y motivación-despersonalización es inversa, mientras que la relación autorrealización-realización personal es directa. Las cuatro dimensiones de la satisfacción laboral explican el $42,4 \%$ de la variabilidad de la dimensión cansancio emocional, $F(4,118)=21,68, \mathrm{p}=0,000$, el 20,5\% de la despersonalización, $F$ $(4,118)=7,60, p=, 000$ y el 30,9\% de la dimensión realización personal, $F(4,118)=13,22, p=0,000$.

En base a los resultados obtenidos se puede concluir que la satisfacción laboral, y cada una de sus dimensiones, son predictores significativos del síndrome de burnout y de cada una de las dimensiones del mismo.

Tabla 2.

Resumen del análisis de regresión múltiple para las dimensiones de los factores extrínsecos e intrínsecos de la satisfacción laboral y el síndrome de burnout.

$\begin{array}{llccr}\begin{array}{l}\text { Dimensiones de } \\ \text { la satisfacción }\end{array} & \text { Burnout } & \text { B } & \beta & \text { IC 95\% } \\ \text { Tarea } & \text { Puntaje bruto } & -0,780 & -0,187^{*} & {[-1,45,-0,11]} \\ \text { Socioorganizativa } & & 0,152 & 0,056 & {[-0,33,0,64]} \\ \text { Motivación } & & -1,533 & -0,289^{* *} & {[-2,48,-0,58]} \\ \text { Autorrealización } & & -1,581 & -0,345^{* * *} & {[-2,43,-0,73]} \\ \text { Tarea } & \text { Cansancio emocional } & -0,722 & -0,369^{* * *} & {[-1,03,-0,41]} \\ & \text { Despersonalización } & 0,019 & 0,011 & {[-0,31,0,35]} \\ & \text { Realización personal } & 0,107 & 0,086 & {[-0,11,0,32]} \\ \text { Socioorganizativa } & \text { Cansancio emocional } & 0,004 & 0,003 & {[-0,22,0,22]} \\ & \text { Despersonalización } & -0,016 & -0,014 & {[-0,25,0,22]} \\ & \text { Realización personal } & -0,165 & -0,204^{*} & {[-0,32,-0,01]} \\ \text { Motivación } & \text { Cansancio emocional } & -0,583 & -0,234^{* *} & {[-1,02,-0,15]} \\ & \text { Despersonalización } & -0,727 & -0,322^{* *} & {[-1,19,-0,26]} \\ & \text { Realización personal } & 0,281 & 0,178 & {[-0,02,0,58]} \\ \text { Autorrealización } & \text { Cansancio emocional } & -0,472 & -0,219^{*} & {[-0,86,-0,08]} \\ & \text { Despersonalización } & -0,355 & -0,182^{*} & {[-0,77,0,06]} \\ & \text { Realización personal } & 0,673 & 0,492^{* * *} & {[0,40,0,94]}\end{array}$

${ }^{*} p \leq 0,05 .{ }^{* *} p \leq 0,01 .{ }^{* * *} p \leq 0,001$

Variables sociodemográficas y laborales y sindrome de burnout. El primer análisis de regresión múltiple, por pasos sucesivos, para conocer el peso predictivo de las variables sociodemográficas consideradas en esta investigación mostró que la única variable que predice significativamente el burnout es la edad de los sujetos $(\beta=-0,189 ; p=0,044)$. Sin embargo, el coeficiente de determinación $\mathrm{R}^{2}$ indicó que esta explica apenas el 3,6\% de la variabilidad del burnout, $F(1,111)=4,13, p=0,044)$, IC 95\% $[-0,87,-0,01]$.

El segundo análisis de regresión mostró que la edad predice significativamente, $F(1,111)=10,61, p=0,001$, IC 95\% [0,08, 0,33], la dimensión de realización personal $(\beta=0,295$; $p=0,001)$, observando que a medida que aumenta la edad aumenta también la realización personal. También se encontró que predice de manera significativa, $F(1,111)=5,22, p=0,024$, IC 95\% [-0,38, -0,03], la dimensión de despersonalización $(\beta=-0,212 ; p=0,024)$, observándose que a mayor edad corresponde menor despersonalización. El coeficiente de determinación $\mathrm{R}^{2}$ indicó que la edad predice el 8,7\% de la realización personal y 4,5\% de despersonalización.

El análisis de regresión múltiple mostró que ninguna de las variables laborales predice significativamente el burnout.

\section{DISCUSIÓN Y CONCLUSIONES}

De acuerdo a los resultados obtenidos, la satisfacción laboral predice significativamente el síndrome de burnout; a medida que los individuos presentan mayores niveles de satisfacción laboral, la incidencia del síndrome de burnout disminuye.

Este hallazgo es consistente con varias investigaciones previas. Por ejemplo, Cherniss, $1980^{14}$, concluyó que uno de los síntomas más frecuentes del síndrome de burnout es la insatisfacción laboral. Grosch y Olsen ${ }^{15}$, que concluyeron que los clérigos, entre otros, sufren del sindrome debido a las condiciones en las que trabajan, padeciendo burnout debido a factores sistémicos externos, como ser la burocracia, el apoyo administrativo deficiente y las dificultades de las condiciones laborales. Jiménez Figueroa et $\mathrm{al}^{16}$ consideraron a la satisfacción laboral como un factor protector frente al burnout, recomendando que se elaboren politicas que fomenten la satisfacción laboral. Otros autores señalaron que la insatisfacción laboral es un factor importante en el desarrollo del síndrome, concluyendo que a menor satisfacción laboral más incidencia de burnout. ${ }^{11}$

La relación más significativa se produjo entre satisfacción laboral y la dimensión de cansancio emocional. Estos resultados coincidieron con los encontrados por Hermosa Rodríguez, Aldrete Rodríguez et al y Gil Monte y Peiró ${ }^{10,17,18}$ que concluyeron que la satisfacción laboral se relaciona con las tres dimensiones del síndrome, pero esta relación es más intensa con el cansancio emocional, mostrando que a mayor satisfacción laboral, menor es el cansancio emocional. Esto puede deberse en parte a la sobrecarga mental y emocional a la que está sometido el docente, y a que dicha sobrecarga se ve amortiguada por los niveles de satisfacción laboral. Pareciera ser que el sentir que la jornada laboral es extensa, que no existen pausas para descansar, que el trabajo es poco flexible, entre otras cosas, generan un agotamiento de las energías y de los recursos emocionales de los docentes. Además, como se mencionó al analizar los resultados, el cansancio emocional es generalmente la primera dimensión que se ve afectada, y al encontrarse la muestra en una fase incipiente de burnout, probablemente es por ello que la satisfacción laboral se relacione más significativamente con dicha dimensión. Se encontró también una relación negativa significativa entre satisfacción laboral y despersonalización, coincidiendo con lo expuesto por Hermosa Rodríguez. ${ }^{10}$ La relación encontrada entre satisfacción laboral y realización personal también ha sido significativa, mostrando que a mayor satisfacción laboral mayor es la realización personal; en este sentido se difiere con Hermosa Rodríguez ${ }^{10}$, que encontró que existe una relación positiva entre satisfacción laboral e ineficacia.

Los resultados mostraron que los factores intrínsecos son los que mejor predicen el sindrome de burnout y cada una de sus dimensiones. A mayor satisfacción intrínseca, menor es el síndrome de burnout, menor es el cansancio emocional y la despersonalización, y mayor la realización personal. Estos resultados coincidieron con Parada et $\mathrm{al}^{9}$, que encontró que la satisfacción intrínseca poseía la mayor fuerza en la correlación con las dimensiones del burnout. Ramírez Pérez y Lee Maturana ${ }^{19}$ concluyeron que los niveles altos de agotamiento emocional en las mujeres de la muestra se explicaban por los bajos niveles de satisfacción intrínseca al igual que los altos sentimientos de despersonalización. Esto podría deberse a que la muestra estudiada posee una fuerte vocación hacia la docencia, evidenciada en el alto porcentaje de docentes que ha contestado que volvería a elegir la docencia como profesión, además de que le brinda las posibilidades de obtener importantes logros y desarrollar sus 
habilidades. Esto podría favorecer la realización personal, ya que sienten que logran resultados a través de su tarea $\mathrm{y}$, a la vez, esto podría permitir que vieran a los alumnos como actores que permiten dichos logros, minimizando así la despersonalización.

Los factores extrínsecos no mostraron predecir el síndrome de burnout como puntaje bruto, aunque los resultados mostraron que predicen significativamente la dimensión de cansancio emocional. Estos resultados ponen de manifiesto la importancia de mejorar la satisfacción intrínseca, cuando en la mayoría de las instituciones los únicos programas que se llevan a cabo tratan de minimizar la insatisfacción, mejorando los factores extrínsecos.

$\mathrm{Al}$ considerar cada una de las dimensiones de los factores de la satisfacción laboral, se encontró que la tarea es la que mejor predice el cansancio emocional, seguida de la dimensión de motivación, y en menor medida por la autorrealización. Esto podría deberse a que, como lo expresa Cornejo $\mathrm{Chávez}^{20}$, se ha intensificado en los últimos años la cantidad y variedad de tareas que deben realizar los docentes, incluyendo responsabilidades que otrora eran propias de las familias. La carga de trabajo normal permite que el docente se adapte, pero la sobrecarga laboral no posibilita que el organismo se recupere de las actividades cotidianas. Según El Sahili González ${ }^{7}$ la sobrecarga laboral genera un problema actitudinal que se ve reflejado en la casi ausencia de recursos creativos por parte del docente en sus clases. La despersonalización fue explicada en mayor medida por la motivación y, en segundo lugar, por la autorrealización. Cuando el docente no tiene en claro los motivos por los que realiza su tarea, esta le parece más rutinaria y agobiante, repercutiendo en el trato y la relación que establece con sus alumnos. Esto podría deberse a que, como lo expresa El Sahili González ${ }^{7}$, la insatisfacción laboral produce desmotivación, falta de interés por el trabajo y apatía, apatía que puede traducirse en una actitud fría y distante hacia los receptores de su trabajo, los alumnos. La autorrealización es la única dimensión que predice significativamente la realización personal; como lo expresan Aguirre et $\mathrm{al}^{21}$, los docentes buscan la autorrealización, expresando frases como "desarrollo personal", "quiero seguir superándome” priorizándola a metas económicas, de prestigio o poder. Sin embargo, las condiciones académicas (indisciplina, problemas de aprendizaje, etc.) y las condiciones económicas (como ser el salario docente) no facilitan la autorrealización, todo ello deriva en burnout, evidenciado en la dimensión de realización personal. Ello podría deberse a que en el nivel medio la cristalización de expectativas se vuelve menos factible y eso facilita la aparición del desgaste laboral. Según El Sahili González ${ }^{7}$, la capacidad de tomar decisiones, intervenir y sentir que tiene dominio sobre su tarea es una de las principales fuentes de estímulo para un docente, constituyendo un protector del desgaste profesional. La dimensión socioorganizativa predijo significativamente la dimensión de realización personal, pero con signo nega- tivo, indicando que a mayor satisfacción socioorganizativa menor es la realización personal, en oposición a todos los demás resultados en los que esta relación es inversa. Esto podría deberse a que la muestra está compuesta por varios docentes jóvenes, que podrian estar satisfechos con la organización y administración, ya que no poseen una trayectoria laboral que les permita evaluar la dimensión socioorganizativa de la institución ni compararla con otras instituciones, pues es su primer trabajo pero, sin embargo, sienten ineficacia y sentimientos de baja realización personal.

En cuanto a la relación de las variables sociodemográficas y laborales y el burnout, se encontraron relaciones significativas únicamente entre la edad y el burnout, por lo que se acepta parcialmente la hipótesis. Los resultados mostraron que a menor edad, mayor es la incidencia del síndrome de burnout, indicando que la edad tiene un efecto moderador sobre el síndrome, especialmente de las dimensiones de realización personal y despersonalización. Los resultados de esta investigación coincidieron con Alcaraz Ramos ${ }^{22}$ en que esto puede deberse a que el docente adquiere mayor seguridad en la tarea que desempeña y esto lo convierte en menos vulnerable al estrés laboral, y con Napione Bergé ${ }^{23}$ que indica que con los años el docente adquiere experiencia, la maduración propia de la edad y pierde una visión irreal de la vida en general, esta visión irreal es la que hace que los docentes jóvenes idealicen la profesión docente $\mathrm{y}$, al no coincidir sus expectativas con la realidad del aula, experimentan una baja realización personal. Cherniss $^{24}$ también concluyó que los profesores más jóvenes son los que presentan mayor vulnerabilidad al burnout, en concordancia con otras investigaciones que indicaron que los profesores más jóvenes experimentan niveles superiores de estrés y de agotamiento emocional y fatiga. ${ }^{25,26}$ Sin embargo, Napione Bergé $^{23}$ concluyó que los profesores que tienen entre 44 y 53 años fueron los más afectados en la dimensión de agotamiento emocional, aunque los mayores de 54 años obtuvieron puntuaciones medias menores que las otras edades.

Teniendo en cuenta la importancia que tiene el trabajo en la vida de los docentes, se recomienda que las instituciones desarrollen programas de intervención y desarrollo para aumentar la satisfacción laboral y la adquisición de competencias que les permitan afrontar los desafíos de la tarea docente, para prevenir el riesgo de que los docentes sufran o desarrollen burnout. Considerando que los factores intrínsecos constituyeron los mejores predictores del sindrome, se recomienda que los programas para aumentar la satisfacción laboral estén orientados a aumentar dicha satisfacción y no a disminuir la insatisfacción extrínseca.

Considerando que son los docentes más jóvenes los que presentaron menor realización personal y mayor despersonalización, se recomienda que se incluya dentro de los planes curriculares de formación de docentes contenidos dirigidos a formar competencias de afrontamiento del estrés docente. 
1. Maslach C, Jackson S. Inventario "burnout" de Maslach. Madrid: TEA; 1997.

2. Oros L, Main M. ESLA-educación: una escala para evaluar la satisfacción laboral de los docentes. Revista Internacional de Estudios en Educación. 2004; 4(1):20-34.

3. Gil-Monte $P_{1}$ Nuñez-Román $E_{1}$ Selva-Santoyo Y. Relación entre el Sindrome de Quemarse por el Trabajo (Burnout) y Sintomas Cardiovasculares: un estudio en técnicos de prevención de riesgos laborales. Interam J Psychol. 2006; 40(2):227-232.

4. Arís Redó N. El Sindrome de Burnout en los docentes. Electronic Journal of Research in Educational Psychology-EJREP [en línea]. 2009 [citado oct 2016]; 7(18): 829-848. Disponible en: http://www.investigacion-psicopedagogica.org/ revista/articulos/18/espannol/Art_18_303.pdf

5. Maslach C, Jackson S. The measurement of experienced burnout. J Organ Behav. 1981; 2(2):99-113.

6. Salazar A. Factores predictores de la satisfacción laboral de las educadoras de Belice. Revista Internacional de Estudios en Educación. 2002; 2(1):23-59.

7. El Sahili González L F. Burnout... y docencia, un recorrido por el sentimiento gradual de desilusión y desgaste en la profesión educativa, sus consecuencias y soluciones. México: Trillas; 2012.

8. Guerrero E. Análisis pormenorizado de los grados de burnout y técnicas de afrontamiento del estrés docente en profesorado universitario. Anal Psicol. 2003; 19(1):145-158.

9.- Parada M, Moreno R, Mejías Z, Rivas A, Rivas F, Cerrada J, et al. Satisfacción laboral y síndrome de burnout en el personal de enfermería del Instituto Autónomo Hospital Universitario Los Andes (IAHULA), Mérida, Venezuela, 2005. Rev Fac Nac Salud Pública. 2005; 23(1):33-45.

10. Hermosa Rodríguez A. Satisfacción laboral y "síndrome de burnout" en profesores de educación primaria y secundaria. Rev Colomb Psicol. 2006;15:81-89.

11. Cavalcante Silva J. Satisfacción en el trabajo de los directores de escuelas secundarias públicas de la región de Jacobina (Bahía-Brasil) [tesis doctoral]. Barcelona: Universidad Autónoma de Barcelona-Facultad de Ciencias de la Educación; 2004.

12. Maslach $C$, Jackson S. Maslach burnout Inventory. $2^{\mathrm{a}}$ ed. Palo Alto: Consulting Psychologists Press; 1986.

13. Oros $L, M a i n, M$, Duarte $P$. Desgaste profesional y satisfacción laboral en una muestra de docentes; Informe final correspondiente al Proyecto $\mathrm{N}^{\circ} 12$ presen- tado a la Secretaría de Ciencia y Técnica de la Universidad Adventista del Plata. Entre Ríos: Universidad Adventista del Plata; 2002.

14. Hein N. Estudio del síndrome de burnout en pastores adventistas y su relación con la satisfacción laboral y caracteristicas de personalidad [tesis]. Entre Rios: Universidad Adventista del Plata; 2005.

15. Grosch W, Olsen D. Clergy burnout: An integrative approach. J Clin Psychol. 2000; 56(5):619-632.

16. Jiménez Figueroa $A$, Jara Gutiérrez $M$, Miranda Celis E. Burnout, satisfacción laboral y apoyo social en docentes. Psicol Escol Educ-ABRAPEE. 2012; 16(1):125-134.

17. Aldrete Rodríguez $M$, Aranda Beltrán $C$, Valencia Abundiz S, Salazar Estrada J. Satisfacción laboral y sindrome de burnout en docentes de secundaria. Revista de Educación y Desarrollo (Guadalajara). 2011; 8(17):5-22.

18. Gil-Monte $P$, Peiró J. Perspectivas teóricas y modelos interpretativos para el estudio del sindrome de quemarse por el trabajo. Anal Psicol. 1999; 15(2): 261-268.

19. Ramírez Pérez $M$, Lee Maturana S. Síndrome de Burnout entre hombres y mujeres medido por el clima y la satisfacción laboral. Polis (Santiago). 2011; 10(30):431-446.

20. Cornejo Chávez R. Entre el sufrimiento individual y los sentidos colectivos: Salud laboral docente y condiciones de trabajo. Docencia. 2008; 35:77-85.

21. Aguirre J, Aparicio M, Marsollier R. Docencia, educación y escuela "en riesgo". Un estudio en docentes de nivel medio de Mendoza. Congreso Iberoamericano de Educación; 13-15 sept. 2010; Buenos Aires, Argentina. Buenos Aires: OEl; 2010.

22. Alcaraz Ramos C. Frecuencia y factores de riesgo asociados al sindrome de burnout en un hospital de segundo nivel [tesis doctoral]. Colima: Universidad de Colima-IMSS; 2006.

23. Napione M. ¿Cuándo se quema el profesorado de secundaria? Buenos Aires: Edics. Díaz de Santos; 2008.

24. Ortega Ruiz C, López Ríos F. El Burnout o Síndrome de estar quemado en los profesionales sanitarios: revisión y perspectivas. Int J Clin Health Psychol. 2004; 4(1):137-160.

25. Schwab R, Iwanicki E. Perceived role conflict, role ambiguity, and teacher burnout. Educ Adm 0. 1982; 18(1):60-74.

26. Yagil D. If anything can go wrong it will: Occupational stress among inexperienced teachers. Int J Stress Manage. 1998; 5(3):179-188. 